\title{
Acquisition de contrôle : le sens fiscal de la notion de contrôle
}

\author{
Éric Bélanger ${ }^{1}$, CPA, CA, MBA, M. Fisc. \\ Université du Québec à Chicoutimi \\ Marc Bachand $^{2}$, CPA, CA, M. Fisc. \\ Université du Québec à Trois-Rivières
}

\section{INTRODUCTION}

\section{L'objectif du présent article sera de traiter de la différence entre le contrôle de fait et le contrôle de droit.}

Plusieurs raisons peuvent motiver une société à en acquérir une autre. Dans un contexte de mondialisation des marchés où les plus gros sont nettement plus avantagés, il n'est pas surprenant d'avoir vu plusieurs acquisitions d'entreprises au cours des dernières années et il ne serait pas improbable d'en voir autant dans les années futures. Par contre, il existera toujours des personnes qui tenteront de faire ce genre de transaction dans le seul but d'en tirer un avantage fiscal. C'est d'ailleurs pour cette raison que le législateur a élaboré de nombreuses règles afin de freiner les ardeurs de ces individus. Dans le jargon, ces règles sont appelées les règles d'acquisition de contrôle.

\section{LA NOTION DE CONTRÔLE}

Nous pourrions nous interroger sur l'utilité d'aborder la notion de contrôle en se disant qu'il s'agit d'un concept assez simple. Pourtant, il est tout à fait pertinent de s'y attarder, car il existe deux types de contrôle. L'un déclenche les règles d'acquisition de contrôle tandis que l'autre n'a aucun impact sur celles-ci.

\subsection{Le contrôle de droit (de jure)}

Une lecture approfondie de la loi nous permet de constater que la notion de contrôle de droit n'est pas définie. Le législateur a préféré laisser le soin à la jurisprudence de se charger de cette tâche. Il devient donc pertinent de prendre le temps d'observer les différentes causes ayant contribué à définir cette notion.
La connaissance de celles-ci devient donc primordiale si une entreprise ne veut pas avoir de mauvaises surprises au niveau fiscal.

Le point de départ des règles d'acquisition de contrôle amène un problème important. Pour conclure à l'application de ces règles, il faut porter une attention particulière à la notion de contrôle. Celle-ci n'est pas totalement précisée dans la Loi de l'impôt sur le revenu. Cette imprécision laisse place à de multiples interprétations. L'objectif du présent article sera de traiter de la différence entre le contrôle de fait et le contrôle de droit. Il mettra de l'avant des critères ou facteurs que la jurisprudence ou l'Agence du revenu du Canada ont établis pour conclure à l'un ou l'autre de ces types de contrôle. Finalement, nous émettrons une opinion quant à l'attitude du législateur.

L'affaire Buckerfield Ltd. est vraiment le point d'ancrage de toutes les causes traitant du sujet. Le juge devait déterminer si certaines sociétés étaient contrôlées par la même personne ou par le même groupe de personnes, ce qui aurait pu permettre d'en arriver à la conclusion que ces sociétés étaient associées. Dans ce cas, une diminution de charges fiscales ne leur aurait pas été accordée. Le juge Jackett en est arrivé à la conclusion que ces sociétés étaient associées, que le mot «contrôle » prenait le sens de contrôle de droit et que l'on devait l'évaluer en fonction du registre des actionnaires ${ }^{3}$. Voici ce que le juge Jackett a dit :

(TRADUCTION) On pourrait sans doute adopter de nombreuses méthodes pour la 
définition du mot «contrôle » figurant dans un texte tel que la Loi de l'impôt sur le revenu. Je suis d'avis cependant que, à l'article 39 de la Loi de l'impôt sur le revenu, le mot «contrôlées » évoque le droit de contrôle auquel donne lieu le titulaire dans l'élection du conseil d'administration. ${ }^{4}$

Nous pouvons résumer les précédents propos en disant qu'une personne qui détient $50 \%$ plus un des droits de vote à une assemblée des actionnaires, apte à élire le conseil d'administration, contrôle ladite société. Cette interprétation a également été utilisée par la Cour suprême du Canada dans la cause Dworkin Furs (Pembroke) Ltd $^{5}$ et autres ${ }^{6}$.

L'affaire Oakfield Developments est également très pertinente. Le juge Judson devait trancher lequel des deux groupes avait le contrôle de la société. L'un des deux groupes détenait l'ensemble des actions ordinaires leur procurant $50 \%$ des droits de vote et l'autre groupe détenait toutes les actions privilégiées qui leur donnait l'autre moitié des votes. Il devient évident que selon le critère de Buckerfield, il est impossible de donner le contrôle à un groupe en particulier. Le juge s'est exprimé de la façon suivante pour rendre sa décision :

(TRADUCTION) Le groupe des dirigeants détenait $50 \%$ des voix, étant propriétaire des actions ordinaires. Il avait droit à tous les superbénéfices lors d'une distribution de dividendes, après le paiement du dividende cumulatif fixe aux actionnaires privilégiés. Advenant la mise en liquidation de Polestar, il avait droit à tout le surplus après le remboursement de capital et le paiement d'une prime de 10 pour cent aux actionnaires privilégiés. Le nombre de voix dont il jouissait était suffisant pour autoriser l'abandon des lettres patentes de la compagnie. À mon avis, ces circonstances suffisent à donner le contrôle au groupe lorsque les propriétaires d'actions privilégiées non participantes détiennent l'autre moitié des voix. ${ }^{7}$

Lorsqu'il y a par ailleurs une impasse à $50 / 50$, nous voyons que ce n'est pas tant le pouvoir d'élire les administrateurs de la société qui a été retenu, mais le pouvoir de dissoudre la société. C'est le nombre de privilèges accordés aux actionnaires ordinaires qui a permis l'attribution du contrôle de la société. Cette cause vient quelque peu nuancer les critères énoncés dans Buckerfield ${ }^{8}$.

La cause Dworkin Furs souligne l'importance majeure que peut jouer une entente contractuelle. L'article 6 des statuts faisait dépendre l'adoption de toute motion au consentement unanime des actionnaires. Le juge Hall a émis les propos suivants :

(TRADUCTION) [...] sans l'article 6 des statuts, Isodore Aaron et Alexander Aaron contrôlaient la société intimée, étant donné qu'ils détenaient 698 des 1008 actions en leurs propres noms avant le 14 juillet 1961, et par la suite au nom de Aaron's (Prince Albert) Limited, société qu'ils contrôlaient également ${ }^{9}$.

Le juge Hall a estimé que la détention de la majorité des actions avec des droits de vote était rendue nulle par l'article 6 des statuts ${ }^{10}$.

L'affaire Vineland Quarries and Crushed Stone Limited vient par contre modifier le critère de Buckerfield. Elle amène la notion de contrôle direct et indirect. Dans cette cause, le juge Cattanach devait déterminer si trois sociétés étaient liées. M. Sauder et M. Thornborrow détenaient la société $\mathrm{n}^{\circ} 1$. M. Thornborrow possédait la société $\mathrm{n}^{\circ} 2$. M. Sauder et la société $\mathrm{n}^{\circ} 2$ contrôlaient la société $n^{\circ} 3$. Le juge en est venu à la conclusion que les trois sociétés étaient liées, même si M. Thornborrow détenait le contrôle de la société $\mathrm{n}^{\circ} 3$ via une personne morale. Le juge Cattanach s'est exprimé de la façon suivante :

(TRADUCTION) Compte tenu de l'affaire British American Tobacco, je ne crois pas qu'il soit acceptable d'examiner les registres des actionnaires de l'appelante et de Sauder and Thornborrow Ltd. et de terminer là l'examen. Il est nécessaire d'examiner les registres des actionnaires de Bold Investments (Hamilton) Limited et de Sauder and Thornborrow Limited, pour savoir si l'appelante et les deux autres sociétés sont contrôlées par le même «groupe de personnes ». Si l'actionnaire inscrit est dans le premier cas une personne morale, il faut aller au-delà du registre des actionnaires ${ }^{11}$. 
Lorsqu'une société est détenue par une deuxième société, il est nécessaire d'aller dans le registre des actionnaires de la deuxième société afin de pouvoir établir qui détient le contrôle de la première société.

Cette cause démontre bien qu'il n'est pas nécessaire de posséder directement les actions d'une société afin d'en avoir le contrôle. Celui-ci peut être exercé de manière indirecte. Donc, lorsqu'une société est détenue par une deuxième société, il est nécessaire d'aller dans le registre des actionnaires de la deuxième société afin de pouvoir établir qui détient le contrôle de la première société. De la même manière, s'il y a acquisition de contrôle de la société mère, il en sera de même pour la filiale ${ }^{12}$.

L'arrêt Vina-Rug, quant à lui, apporte la notion de groupe de personnes. Le juge Abbott devait déterminer si deux sociétés étaient associées pour savoir si elles pouvaient profiter d'un allègement fiscal. La totalité des actions de l'une des sociétés était détenue par un père, ses deux fils et une quatrième personne. La seconde société était détenue par les deux fils et ce même quatrième individu. Le juge Abbott a conclu que les deux sociétés étaient contrôlées par le même groupe de personnes. Voici comment il s'est exprimé :

(TRADUCTION) Le juge de première instance a conclu que John Stradwick Jr., W.L. Stradwick et H.D. McGilvery, qui collectivement détenaient plus de $50 \%$ des actions de Stradwick's Limited, avaient à toutes les époques pertinentes un lien suffisant pour être en état d'exercer un contrôle ${ }^{13}$.

Il en ressort que des actionnaires qui se regroupent et qui sont en mesure d'exercer un contrôle majoritaire sont rattachés par un lien suffisant pour l'application du contrôle du jure et ceux-ci contrôlent la société. Il n'est pas nécessaire que le groupe exerce le contrôle, mais qu'il soit en état de l'exercer ${ }^{14}$.

Finalement, 1'affaire Duha Printers (Western) Ltd. apporte une belle conclusion à la notion de contrôle de jure. Cet arrêt est très important, car le juge Iacobucci met de l'avant les critères afin de pouvoir conclure au contrôle de droit en intégrant l'ensemble de la jurisprudence. De ses propos découlent les principes suivants :

\section{(TRADUCTION)}

1) Le paragraphe 111(5) de la Loi de l'impôt sur le revenu vise le contrôle de jure, et non pas le contrôle de facto.

2) Le critère général du contrôle de jure a été énoncé dans l'arrêt Buckerfield's, précité : il s'agit de décider si l'actionnaire majoritaire exerce un « contrôle effectif » sur « les affaires et les destinées » de la société, contrôle qui ressort de la «propriété d'un nombre d'actions conférant la majorité des voix pour l'élection du conseil d'administration ».

3) Pour décider s'il y a «contrôle effectif », il faut prendre en considération ce qui suit :

a. La loi qui régit la société;

b. Le registre des actionnaires de la société;

c. Toute restriction, particulière ou exceptionnelle, imposée soit au pouvoir d'actionnaire majoritaire de contrôler l'élection du conseil, soit au pouvoir du conseil de gérer l'entreprise et les affaires internes de la société, qui ressort de l'un ou l'autre des documents suivants :

i. Des actes constitutifs de la société;

ii. D'une convention unanime des actionnaires

4) Les documents autres que le registre des actionnaires, les actes constitutifs et les conventions unanimes des actionnaires ne doivent généralement pas être pris en considération à cette fin.

5) Lorsqu'il existe une restriction du genre visé à l'alinéa 3c), l'actionnaire majoritaire peut tout de même exercer le contrôle de jure, à moins qu'il ne dispose d'aucun moyen d'exercer un « contrôle effectif » sur les affaires et les destinées de la société, d'une manière analogue ou équivalente au critère de Buckerfield' $\mathrm{s}^{15}$. 
L'Agence du revenu du Canada a admis être liée par cette décision de la Cour suprême du Canada ${ }^{16}$.

Il peut être intéressant à ce stade de se questionner sur la définition d'une convention unanime entre des actionnaires. Effectivement, cette expression revient à plusieurs reprises dans la notion du contrôle de jure. Il s'agit d'un contrat entre les divers actionnaires d'une société. Son utilité vise surtout à prévenir ou aider à gérer les conflits. Elle contient normalement plusieurs clauses que les actionnaires auront jugées importantes. Par exemple, on pourrait en retrouver une qui traite de l'achat-vente des actions, du droit de premier refus, de l'évaluation de la société, etc. Cependant, il faut bien différencier une convention unanime d'une convention non unanime.

La convention unanime, comme tout contrat, lie les personnes qui l'ont signée, c'est-à-dire les actionnaires. Cependant, contrairement à une convention non « unanime», la loi lui reconnaît un caractère contraignant exceptionnel, exorbitant même, car la convention unanime lie à la fois les administrateurs, la compagnie, et les nouveaux actionnaires (tant les souscripteurs d'actions que les cessionnaires d'actions) ${ }^{17}$.

\subsection{Le contrôle de fait (de facto)}

Avant d'aborder ce thème, il faut bien comprendre certaines subtilités. Ce type de contrôle ne déclenche pas les règles d'acquisition de contrôle. Il est important de l'aborder afin de bien le différencier du contrôle de droit. Par contre, nous devons bien réaliser qu'il ne s'applique pas dans un contexte d'acquisition de contrôle, contrairement au contrôle de droit.

L'étude du contrôle de jure nous a démontré que nous devions procéder à une analyse approfondie des documents constitutifs de la société. Une personne avertie pourrait tenter de manipuler ces documents afin d'éviter le contrôle de droit, ce qui irait à l'encontre de l'objet et de l'esprit de la Loi. Donc, pour pallier à ce risque, en 1988, le législateur a élargi la notion de contrôle à celle du contrôle de fait ${ }^{18}$.

Pour le différencier du contrôle de droit, le législateur a mentionné que lorsque l'expression «contrôlée directement ou indirectement de quelque manière que ce soit » est utilisée dans la Loi de l'impôt sur le revenu, cela veut dire que l'on fait référence à la notion de contrôle de facto.

L'Agence du revenu du Canada a cru bon d'apporter certaines spécifications au sujet du contrôle de fait :

Le contrôle de fait va au-delà du contrôle de droit et comporte la capacité de contrôler « de fait » en exerçant une influence directe ou indirecte. On peut détenir le contrôle de fait sans même posséder d'actions. Le contrôle de fait peut se traduire, par exemple, par le pouvoir que possède une personne de changer le conseil d'administration ou de revenir sur les décisions de celui-ci, de prendre d'autres décisions concernant les actions de la société à court, à moyen ou à long terme, de mettre fin directement ou indirectement à la société ou à son entreprise ou de s'en approprier les bénéfices ou les biens. Même si elle n'est pas exercée effectivement, l'existence d'une telle influence serait suffisante pour conférer le contrôle de fait $^{19}$.

Afin de nous aider à y voir plus clair, l'ARC a mis de l'avant certains éléments pour conclure au contrôle de facto :

a) Le pourcentage de propriété des actions avec droit de vote (lorsqu'il n'est pas supérieur à $50 \%$ ) par rapport aux actions que les autres actionnaires détiennent;

Il pourrait s'agir d'un individu qui détient un bloc de $30 \%$ des actions tandis qu'aucun autre actionnaire n'en détient plus de $5 \%$. Cet actionnaire du bloc de $30 \%$ détiendrait probablement le contrôle de fait de la société.

b) La propriété d'une importante dette d'une société qui peut devenir payable sur demande (à moins d'être assujettie à une exemption en vertu du paragraphe $256(3)$ ou (6)) ou d'un placement important dans des actions privilégiées rachetables au gré du détenteur; 
Les paragraphes $256(3)$ et 256(6) sont des situations où deux sociétés cesseraient d'être associées. Par exemple, pour le paragraphe 256(3), il faut que deux éléments soient présents. Une société doit détenir le contrôle de fait sur une autre en raison d'un titre de créance dont une partie ou la totalité du principal est impayée à un moment donné. De plus, cette même société doit avoir une convention ou un arrangement exécutable qui stipule qu'à la réalisation d'un évènement dont il est raisonnable de s'attendre, le contrôle de fait cessera d'être exercé. Dans cette situation bien précise, si l'évènement annule la créance due, le contrôle de fait sera réputé non présent tout au long de l'année. Le même genre de logique s'applique pour le paragraphe $256(6)$.

c) Les conventions entre actionnaires prévoyant une voix prépondérante;

Par exemple, si le président de l'assemblée des actionnaires est doté d'une voix prépondérante en cas d'égalité dans les votes, cette convention confère au détenteur de ce privilège un contrôle de fait sur la société.

d) Les relations commerciales ou contractuelles de la société, par exemple, la dépendance économique à l'endroit d'un fournisseur ou d'un client unique;

e) La possession de connaissances tout à fait particulières qui sont requises pour l'exploitation de l'entreprise;

Cette situation survient lorsqu'une personne possède des informations qu'elle est la seule à détenir. Si un ingénieur est

\section{CONCLUSION}

Il est curieux que le législateur n'ait pas pris le temps de définir le contrôle de droit. Il est évident, qu'au moment de la rédaction des articles de loi, celui-ci avait une idée, peut-être floue, mais tout de même une tangente qu'il voulait donner à cette définition. En laissant le soin aux tribunaux de faire le seul à connaître le fonctionnement d'une machine, que cette machine est primordiale dans la production et que la formation d'un remplaçant prendrait plusieurs mois, cet ingénieur exercerait un contrôle de fait sur l'usine.

f) L'influence qu'un membre d'une famille, qui est un actionnaire, un créancier, un fournisseur, etc., d'une société peut avoir sur un autre membre de la famille qui est un actionnaire de la société ${ }^{20}$.

En plus des facteurs énumérés ci-haut, nous pourrions ajouter la composition du conseil d'administration et le contrôle au jour le jour de la gestion de l'entreprise.

En résumé, le contrôle de fait a lieu à un moment précis et il n'est pas nécessaire de l'avoir tout au long de l'année d'imposition. Il résulte très souvent d'une influence et il peut exister même si aucune action n'est détenue dans la société.

Depuis la venue du paragraphe 256(5.1) de la LIR en 1988 sur la notion du contrôle de fait, les décisions ayant fait jurisprudence en matière de contrôle de fait sont très rares. Par contre, certaines décisions sur le contrôle de droit ont mené à la conclusion qu'il s'agissait plutôt d'un contrôle de facto. Par exemple, dans l'affaire Dworkin Furs, une voix prépondérante en cas d'égalité lorsque nous sommes président du conseil d'administration à l'assemblée des actionnaires et des administrateurs est considérée comme un contrôle de fait et non de jure ${ }^{21}$.

\section{Le contrôle de fait a lieu à un moment précis et il n'est pas nécessaire de l'avoir tout au long de l'année d'imposition.}

ce travail, le législateur n'avait aucune assurance que la décision qui serait prise correspondrait à ce qu'il souhaitait au moment de la rédaction.

De plus, cela complique énormément le travail des fiscalistes qui font des planifications pour 
leurs clients. Pour en arriver à l'application des règles d'acquisition de contrôle, il faut conclure à la notion de contrôle de droit. Donc, dans les premiers temps, lorsque la jurisprudence n'était pas suffisamment étoffée, il y avait toujours un risque que la planification proposée au client soit refusée par l'Agence du revenu du Canada et qu'elle fasse jurisprudence dans le domaine. Par conséquent, pour les cabinets comptables et les fiscalistes, il devenait très difficile de proposer ce genre de mandat aux clients, car il fallait toujours tenir compte d'un risque lié à une jurisprudence en évolution. À ce titre, la prévisibilité du traitement accordé en fonction de la Loi demeure un concept fondamental à atteindre en impôt.

\section{Nous avons démontré que le législateur a introduit la définition du contrôle de fait en 1988, car il craignait que certains individus tentent de manipuler les documents constitutifs des sociétés.}

Par contre, il est paradoxal de penser que ce sont justement ces zones grises, plus ou moins définies par la Loi de l'impôt sur le revenu, qui permettent de faire de belles planifications fiscales. Sans elles, les fiscalistes auraient beaucoup moins de latitude dans leurs planifications et le département de fiscalité à l'intérieur des cabinets comptables aurait un rôle beaucoup moins important.

Nous avons démontré que le législateur a introduit la définition du contrôle de fait en 1988, car il craignait que certains individus tentent de manipuler les documents constitutifs des sociétés. L'apparition de cette définition résulte d'une réaction à la définition globale que la jurisprudence a faite du contrôle de droit depuis plusieurs années. Donc, plutôt que d'adopter une position proactive en apportant des définitions adéquates qui démontrent clairement ses intentions, le législateur préfère adopter une attitude réactive lorsqu'il n'est pas tout à fait satisfait du résultat donné par les tribunaux. Du point de vue d'un fiscaliste qui veut faire des planifications fiscales pour ses clients, cette attitude du législateur est tout à fait déplorable.

\section{BIBLIOGRAPHIE ET NOTES}

1'Éric Bélanger est professeur de fiscalité à l'Université du Québec à Chicoutimi.

${ }^{2}$ Marc Bachand est professeur de fiscalité à l'Université du Québec à Trois-Rivières.

${ }^{3}$ Auger, François, «Impact des règles d'acquisition de contrôle », dans Colloque 103 - Achat/Vente d'une PME : Planification fiscale pour l'acheteur et le vendeur, Montréal, Association de planification fiscale et financière, 2001.

${ }^{4}$ Bukerfield's Limited v. MNR, 64 D.T.C. 5301, Cour de l'Échiquier.

${ }^{5}$ MNR v. Dworkin Furs (Pembroke) Ltd. et al., 67 D.T.C. 5035, Cour suprême du Canada.

${ }^{6}$ Morin, Philippe-Antoine, «Incidence des conventions entre actionnaires sur le contrôle des sociétés et sur les liens entre entités » dans Colloque 166 - Planification successorale, relève d'entreprise et conventions entre actionnaires, Montréal, Association de planification fiscale et financière, 2007.

${ }^{7}$ Oakfield Developments (Toronto) Limited v. MNR, 69 D.T.C. 5175, Cour suprême du Canada.

${ }^{8}$ Supra note 3.

${ }^{9}$ Supra note 5 .

${ }^{10}$ Supra note 3.

${ }^{11}$ Vineland Quarries and Crushed Stone Ltd. v. MNR, 66 D.T.C. 5092, Cour de l'Échiquier.

${ }^{12}$ Supra note 3.

${ }^{13}$ Vina-Rug Canada Ltd. v. MNR, 68 D.T.C. 5021, Cour suprême du Canada.

${ }^{14}$ Supra note 3.

${ }^{15}$ Duha Printer (Western) Ltd. C Sa Majesté la Reine, 98 D.T.C. 6334, Cour suprême du Canada.

${ }^{16}$ Supra note 3.

${ }^{17}$ Paul et Luc Martel (2011). Les conventions entre actionnaires. Une approche pratique, $10^{\mathrm{e}}$ édition, Édition Wilson \& Lafleur.

${ }^{18}$ Supra note 6.

${ }^{19}$ Agence du revenu du Canada, Bulletin d'interprétation IT-64R4 (consolidé), « Sociétés : Associations et contrôle » (13 octobre 2004), par. 21. ${ }^{20}$ Ibid. par. 23.

${ }^{21}$ Lefebvre, Paul A. et Roger Taylor, «Contrôlée directement ou indirectement, de quelque manière que ce soit » dans Congrès 2002, Montréal, Association de planification fiscale et financière. 